\title{
Recurso Educativo Abierto elaborado con GeoGebra para la enseñanza-aprendizaje del principio de Arquímedes
}

\author{
Roberto Téllez Vargas ${ }^{1}$, Sergio Rubio-Pizzorno ${ }^{2}$ \\ ${ }^{1}$ Escuela Emperador Cuauhtémoc - Puebla - México \\ ${ }^{2}$ Instituto GeoGebra Internacional - México/Chile \\ vargastellez1968@gmail.com, sergio.rubio@cinvestav.mx
}

\begin{abstract}
In physics teaching, experimentation with material resources or digital simulations is usual. Both types of resources pose problems for teachers when it comes to using them in their classes, be it cost, availability or impossibility to modify. In response to this scenario, this article presents the development of an educational design that incorporates easily accessible material resources, GeoGebra software for teachers to create simulations, and GeoGebra authoring tools to articulate material and digital resources in an open educational design. All this under the methodological proposal of the Negotiation Process, framed in the Design Based Research.
\end{abstract}

Resumen. En la enseñanza de la física es usual la experimentación con recursos materiales o con simulaciones digitales. Ambos tipos de recurso plantean problemáticas para los profesores a la hora de usarlos en sus clases, ya sea de costo, disponibilidad o imposibilidad para modificar. En respuesta a este escenario, en este artículo se presenta la elaboración de un diseño educativo que incorpora recursos materiales de fácil acceso, el software GeoGebra para que los profesores creen simulaciones, y las herramientas de autor de GeoGebra para articular los recursos materiales y digitales en un diseño educativa abierta. Todo esto bajo la propuesta metodológica del Proceso de Negociación, enmarcada en la Investigación Basada en el Diseño.

\section{Introducción}

En la enseñanza de la física se recurre de manera frecuente a la experimentación con recursos materiales. Sin embargo, algunas veces estos materiales son de difícil acceso tanto por su costo monetario o disponibilidad.

Como respuesta a esta situación, han cobrado popularidad los simuladores digitales, tales como los de PhET [University of Colorado, 2019]. Sin embargo, a pesar de la calidad de tales simulaciones, estas no pueden ser modificadas o, incluso, elaboradas desde cero, por los profesores.

Ante este contexto, en este artículo se plantea la posibilidad de elaborar diseños educativos para la enseñanza de la física, que permita responder a los contextos educativos locales y a la disponibilidad de recursos materiales de la comunidad. Así también, se utiliza el software de matemática dinámica de GeoGebra para sortear la barrera de las simulaciones digitales cerradas, que no permiten modificación, para crear 
simulaciones físicas a la medida de las necesidades de los profesores. Sumado a lo anterior, se explora el uso de las herramientas de autor de GeoGebra, a saber Actividad y Libro GeoGebra, para articular el uso de recursos materiales y digitales con un propósito educativo determinado.

Todo esto para la elaboración de un diseño educativo que permita abordar la noción física del principio de Arquímedes.

\section{Fundamentación teórica}

Al pensar en un aula de clases podemos reconocer que es un espacio educativo especial, donde se relacionan profesores, estudiantes, objetos materiales, así como los diseños educativos, discursos del profesor y la interacción entre todos estos participantes. Dicho en palabras de Cobb et al. (2003), el aula de clase corresponde a una ecología educativa descrita como "un sistema complejo e interactivo que involucra múltiples elementos de diferentes tipos y niveles" (p. 9).

En esta sintonía con esta conceptualización del aula de clase, Rubio-Pizzorno (2018) se refiere a la constitución material de los ambientes educativos reconociendo que:

Ya no son espacios discretos y aislados unos de otros, sino que se construyen de manera simbiótica aprovechando lo mejor de cada uno. A este tipo de ambientes o ecosistemas educativos los denominamos híbridos, puesto que se constituyen a partir de soportes materiales de diversas naturalezas (p. 112).

De esta manera, es importante reconocer que el aula de clase, como un ecosistema educativo híbrido, está constituido por espacios de distinta naturaleza (e.g. digital, material), en los cuales están presente recursos que nos permiten acceder a lo digital o a lo material, específicamente al elaborar un diseño educativo. De esta manera "[es importante] atender a los ambientes de diseño considerando su constitución híbrida, poniendo atención, por un lado en lo específico de cada espacio, y por otro, a las formas de articularse entre los diferentes espacios" [Rubio-Pizzorno, 2018, p. 113].

En este trabajo se retoman las ideas sobre los ecosistemas educativos híbridos para guiar la selección de los recursos usados en la elaboración del REA para abordar el principio de Arquímedes, atendiendo a la disponibilidad de tales recursos en las aulas de clase de la escuela Emperador Cuauhtémoc del estado de Tlaxcala (México), correspondientes al nivel medio-superior de México (Educación Media). Además se la integración de tecnologías digitales abiertas, específicamente el software GeoGebra y los ambientes de creación de REA (o herramientas de autor) de GeoGebra, es decir, Actividad y Libro.

\section{Metodología}

En este trabajo se emplea una metodología de Investigación Basada en el Diseño, la cual propone la elaboración de un diseño educativo para ser implementado con 
VIII Congresso Brasileiro de Informática na Educação (CBIE 2019)

Anais dos Workshops do VIII Congresso Brasileiro de Informática na Educação (WCBIE 2019)

estudiantes, y posteriormente analizar la implementación con el fin obtener elementos para realizar sucesivos rediseños. En palabras de Swan (2014):

La investigación basada en el diseño es un enfoque formativo de la investigación, en el cual un producto o proceso (o "herramienta") es concebido, diseñado, desarrollado y refinado a través de ciclos de promulgación, observación, análisis y rediseño, con la retroalimentación sistemática de los usuarios finales.

(...) La teoría educativa se utiliza para orientar el diseño y el refinamiento de las herramientas y se perfecciona durante el proceso de investigación.

(...) En última instancia, la meta es transformadora; se busca crear nuevas posibilidades de enseñanza y aprendizaje y estudiar su impacto en maestros, niños y otros usuarios finales (p. 148).

De manera específica, en este artículo se reporta la fase de elaboración del diseño educativo, para la cual se siguió la propuesta metodológica de Rubio-Pizzorno (2018) llamada Proceso de Negociación -enmarcada en la corriente de Investigación Basada en el Diseño- el cual funciona como marco para la búsqueda de consensos entre diferentes polos que intervienen en la elaboración de diseños educativos, tales son, (1) los resultados de investigación disciplinares, (2) los saberes docentes [Mercado, 2002] expresados como la experiencia docente desarrollada a través del ejercicio de la docencia, y (3) la atención a los ecosistemas educativos híbridos.

El Proceso de Negociación se realizó en el marco del Diplomado "Diseño de estrategias y recursos educativos", dictado por el Maestro Sergio Rubio-Pizzorno en el periodo de primavera 2019 en el Instituto Nacional de Astrofísica Óptica y Electrónica (INAOE) del estado de Puebla (México).

El Diplomado tiene como objetivo que los profesores puedan elaborar un diseño educativo para un contenido que cada grupo de profesores escogió, según los contenidos que imparten en sus clases. Es decir, se busca que el producto a realizar durante el Diplomado aporte a la realidad docente que cada profesor.

Durante las etapas del Diplomado se fueron realizando sucesivos Procesos de Negociación en los cuales participan todos los miembros del Diplomado, es decir, los profesores en calidad de alumnos y el profesor que lo imparte. Entre los profesores-estudiantes, pasaban por equipos a compartir los avances de su diseño educativo, para recibir la retroalimentación de parte del resto del grupo, la cual se daba de manera verbal y luego se registraba por escrito quedaba en el Grupo GeoGebra dedicado al Diplomado. A continuación, cada equipo realizaba modificaciones a su diseño con base en la retroalimentación que el grupo le entregó.

De es manera se puso en funcionamiento el Proceso de Negociación en sucesivas etapas. En primera instancia para discutir el objetivo del diseño, los objetivos específicos y el proceso hipotético de aprendizaje, como parte de la planificación del diseño, cuya estructura se basa en la Trayectoria Hipotética de Aprendizaje propuesta por Simon (1995). A continuación para reflexionar sobre las versiones del diseño que se estaba elaborando con las Actividades y el Libro GeoGebra. Finalmente, se realiza un pilotaje del diseño educativo utilizando la estrategia de juego de roles, donde profesores 
participan como estudiantes, y la estrategia en práctica, donde los profesores actúan como participantes reflexivos [Grugeon y otros, 2010] para dar retroalimentación de la implementación del diseño.

Un aspecto interesante que aportó en gran medida al polo de experiencia docente del Proceso de Negociación, fue que los participantes del Diplomado son profesoras y profesores de diferentes niveles educativos, que imparten diferentes asignaturas (biología, física, matemáticas, filosofía, entre otras).

Es importante mencionar que al producto desarrollado mediante la puesta en funcionamiento del proceso de negociación corresponde a un diseño educativo que se elaboró usando las herramientas de autor de GeoGebra, Actividades y Libros. Por defecto, todos los recursos que se creen en esos ambientes tienen una licencia Creative Commons Atribución-CompartirIgual 3.0 (CC BY-SA 3.0), la cual permite compartir y adaptar el recurso de manera abierta. Por esta razón es que el diseño educativo elaborado también es posible denominarlo un recurso educativo abierto o simplemente REA.

En términos generales un REA corresponde a:

Cualquier recurso educativo (incluso mapas curriculares, materiales de curso, libros de estudio, streaming de videos, aplicaciones multimedia, podcasts y cualquier material que haya sido diseñado para la enseñanza y el aprendizaje) que esté plenamente disponible para ser usado por educadores y estudiantes, sin que haya necesidad de pagar regalías o derechos de licencia [UNESCO, 2015].

Con base en esta caracterización realizada por la UNESCO es que, con toda seguridad, nos referimos al diseño didáctico elaborado usando Actividad y Libro GeoGebra, como un REA. Es importante señalar que durante el escrito se utiliza tanto diseño educativo o REA para referirse al producto construido para la enseñanza-aprendizaje del principio de Arquímedes.

\section{Elaboración del REA para la enseñanza-aprendizaje del principio de Arquímedes}

Tomando en cuenta tanto las necesidades actuales de los estudiantes y las herramientas tecnológicas de las que se disponen, se ha pensado en la elaboración de este REA, de tal forma que sea utilizado por los docentes en su quehacer diario, así como por los mismos estudiantes, ya sea en su aprendizaje directo o en la complementación y reforzamiento del mismo. Las herramientas de autor que se utilizaron para elaborar el REA corresponden a la Actividad y el Libro GeoGebra. De manera general se elabora un Libro GeoGebra que es el REA completo, el cual está constituido por capítulos y páginas, donde cada una de estas últimas corresponden a una Actividad GeoGebra. Así, el REA para la enseñanza-aprendizaje del principio de Arquímedes corresponde a un Libro GeoGebra de seis capítulos, tal como se muestra en la Figura 1, donde en cada capítulo se abordan temáticas necesarias para realizar un proceso de construcción del principio de Arquímedes a partir de sus componentes, como la densidad, peso, masa, volumen, entre otros. 


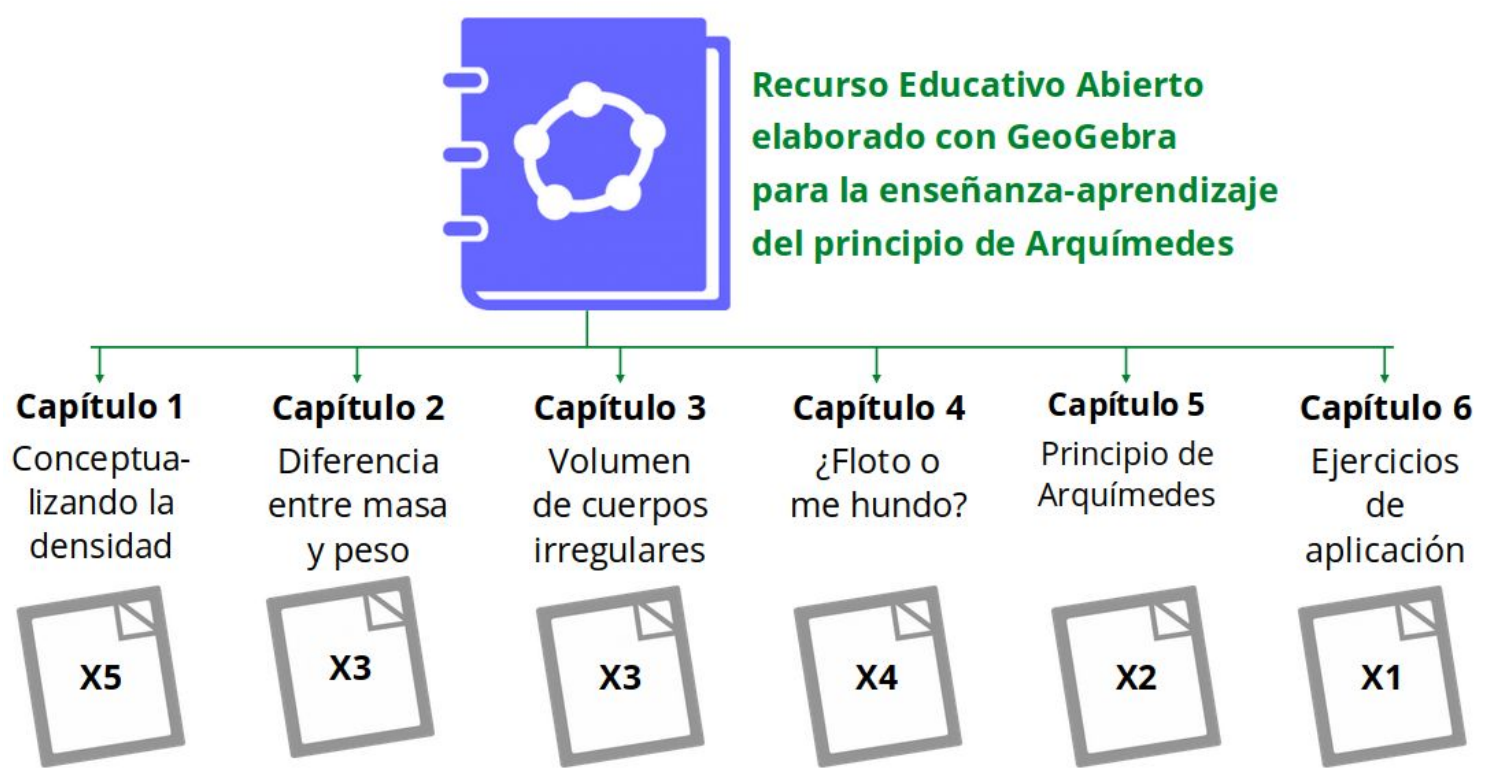

Figura 1. Estructura del REA elaborado en con un Libro y Actividades GeoGebra.

Si bien el REA está elaborado en un Libro GeoGebra, que corresponde a una herramienta digital, también se hace uso de recursos materiales, como semillas, huevos, algodón, entre otros. Esto, en respuesta al reconocimiento de los Ecosistemas Educativos Híbridos y los materiales representativos de cada espacio. La articulación de todos los recursos, ya sean físicos o digitales, se realiza en el Libro GeoGebra, donde se dan las indicaciones para trabajar con los recursos materiales mostrando ejemplos a través de fotos, y se dispone de recursos digitales como animaciones, imágenes, preguntas de respuesta abierta o de opción múltiple, con los cuales se trabaja directamente en el Libro.

Para ilustrar los tipos de tareas que se diseñaron, a continuación se presentan las actividades más representativas del REA, el cual se puede revisar íntegramente en Téllez (2019).

\subsection{Saturación de semillas con recursos materiales}

Sobre un cartón se colocan 100 semillas (ver Figura 2), las medidas del cartón que sirven de base, se van reduciendo hasta tener el área más pequeña, observamos cómo quedan distribuidas las semillas, también se puede calcular su saturación a medida que se reduce el tamaño de la base.
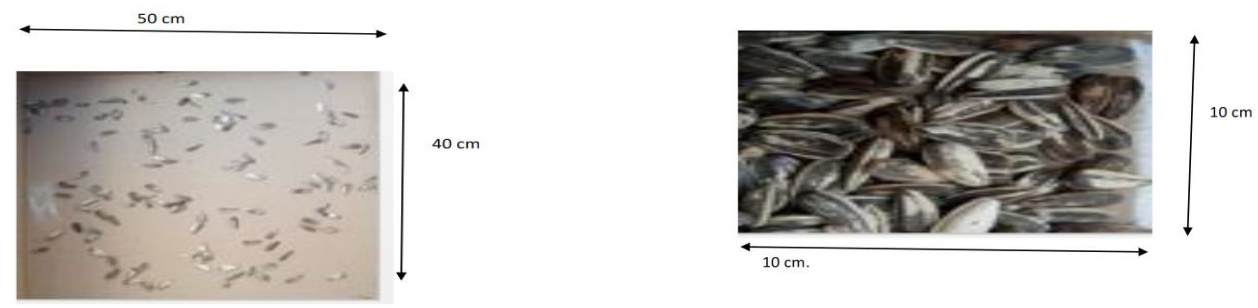
VIII Congresso Brasileiro de Informática na Educação (CBIE 2019)

Anais dos Workshops do VIII Congresso Brasileiro de Informática na Educação (WCBIE 2019)

Figura 2. Saturación de semillas en un área determinada.

\subsection{Saturación de semillas (partículas) con applet GeoGebra}

En un applet GeoGebra se presentan una serie de puntos y por medio de un deslizador se va modificando en área de la figura, lo cual permite observar cómo se van amontonando o dispersando los puntos sobre de ella (ver Figura 3).
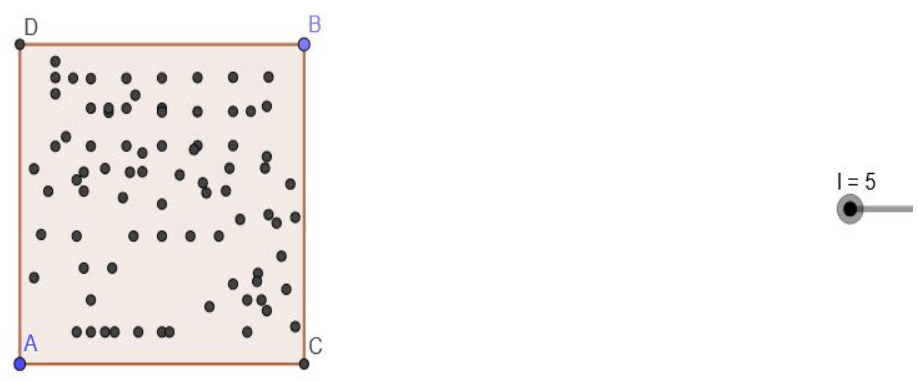

Figura 3. Saturación de semillas (partículas) con applet GeoGebra

\subsection{Relación entre masa y área}

Se muestra la Figura 4 a los alumnos y se pide que determinen el valor de la masa que soporta, el suelo; considerando que el espacio no varía ya que es un cuadrado de $4 \mathrm{~m}$ de cada lado, en todos los casos y otorgando la masa promedio de las personas de $80 \mathrm{~kg}$. Para que con ello visualicen cómo varía la masa sobre el área en cuestión y sobre todo como se incrementa al incrementar en número de personas, en consecuencia la masa.
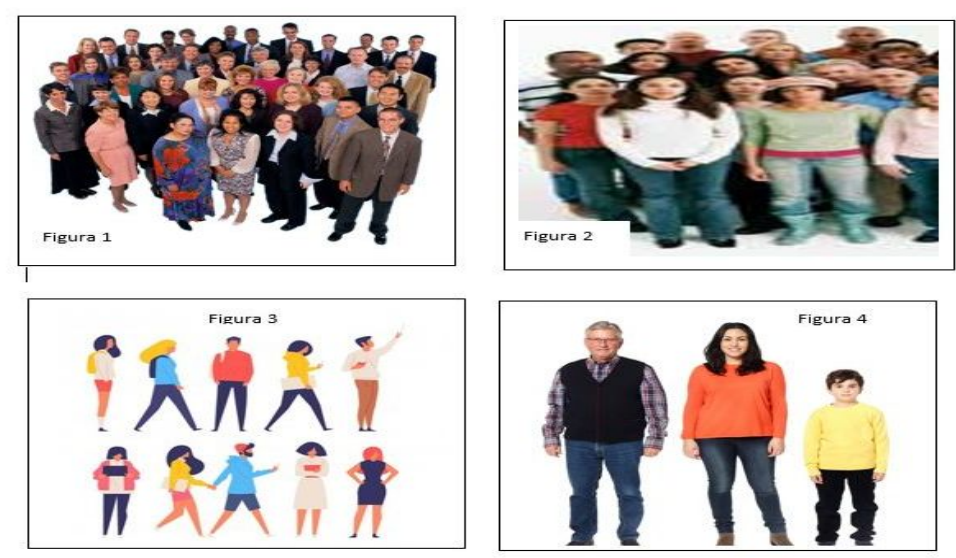

Figura 4. Relación de la masa sobre un área determinada.

\subsection{Relación entre masa y área}

Se pretende que el estudiante relacione el valor de la concentración que se tiene en dos recipientes de la misma masa, pero el volumen que ocupan es diferente, aunque el del recipiente sigue siendo el mismo, como se muestra en la Figura 5, y con ello lanzar una serie de preguntas para que se comprenda la concentración que tiene el recipiente, e irlos llevando al concepto de densidad. 

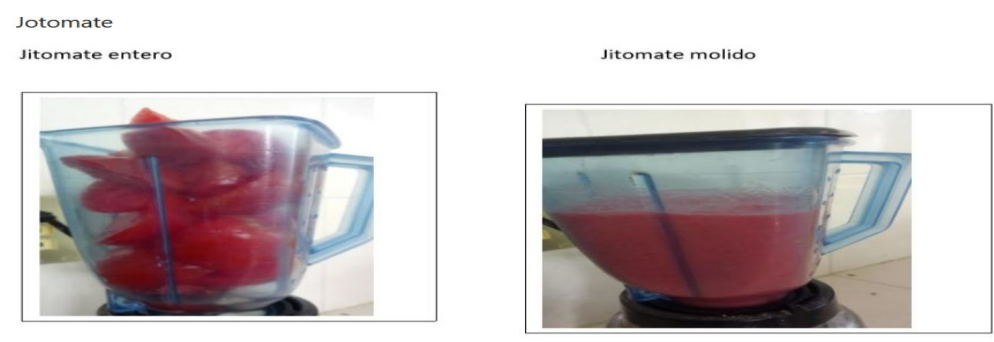

Figura 5. Relación de la masa sobre un volumen determinado.

\subsection{Experimento sobre la densidad y el empuje}

Utilizando 3 vasos transparentes, 1 litro de agua, 1 huevo, 1 cuchara sopera, $200 \mathrm{~g}$ de sal, se realiza un experimento para estudiar la relación entre la densidad del agua, cuando está pura o con sal, y el empuje de esta respecto de un objeto al interior de los vasos, que en este caso corresponde a un huevo.

El experimento se realiza vertiendo $3 / 4$ partes de agua en dos vasos. En uno de ellos se introduce el huevo, observando que éste se ha sumergido hasta el fondo del vaso (Figura 6 - izquierda). En el segundo se agregan 3 cucharadas de sal y se mezcla con el agua, para luego introducir al vaso el mismo huevo de la situación anterior. En este caso, se observa que el huevo flota (Figura 6 - centro).

Dado los dos casos anteriores, se propone el desafío a los estudiantes que en el tercer vaso combinen la cantidad apropiada de agua pura (vaso 1) y agua con sal (vaso 2) para que al sumergir el huevo, este quede flotando a la mitad del vaso. Para ello, en el tercer vaso se agrega la mitad del agua potable del primer vaso y un cuarto del segundo vaso que contiene agua, y a continuación se sumerge el huevo. En caso que este no quede a la mitad del vaso, se va agregando agua con una cuchara hasta lograr la posición deseada (Figura 6 - derecha).
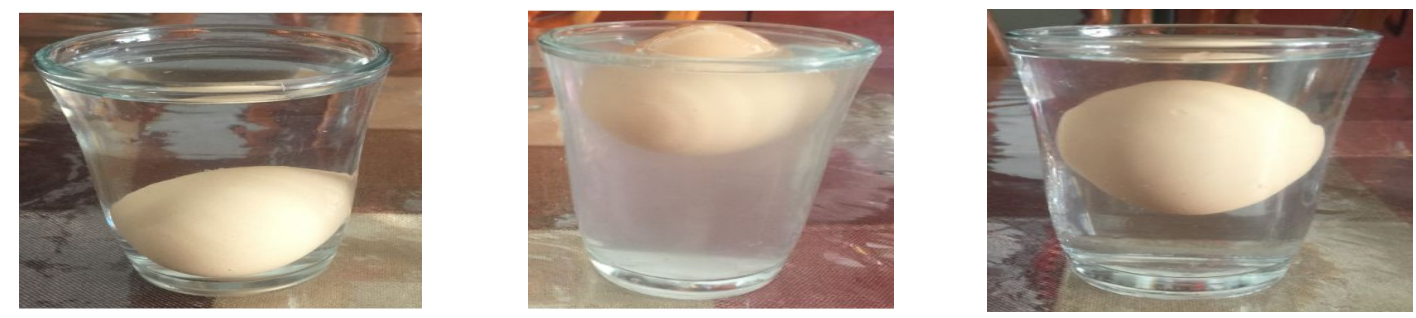

Figura 6. Experimento sobre densidad y empuje.

A continuación se plantean una serie de preguntas con relación al experimento, para que los estudiantes puedan explicar las razones del comportamiento de los tres casos, usando los conceptos ya trabajados en el REA. 


\section{Conclusión y prospectivas}

Como parte de una Investigación Basada en el Diseño, el presente artículo reporta la fase de diseño del REA para la enseñanza-aprendizaje del principio de Arquímedes, que ponga en juego en su elaboración y producto final las ideas de los Ecosistemas Educativos Híbridos. Esto con la intención de acercarse de manera más situada a contextos escolares reales de enseñanza de la física, atendiendo a los recursos (digitales y materiales) disponibles y a las posibilidades que brindan las tecnologías digitales libres, tales como el software o las Herramientas de Autor de GeoGebra.

Por ejemplo, en el REA se articula el uso de semillas en un área determinada y de un applet GeoGebra como simulación de tal situación, con el objetivo de establecer un modelo que permita estudiar la situación física, tanto en casos reales como en casos hipotéticos. O también, usar el ambiente de matemática dinámica de GeoGebra para realizar una explicación conceptual de un experimento realizado con recursos materiales.

Así también el presente trabajo tiene como prospectiva la implementación del REA con estudiantes (fase de implementación) y el posterior análisis de los datos obtenidos de tal implementación (fase de análisis), para así completar un primer ciclo de la metodología Investigación Basada en el Diseño, que dote de elementos para realizar un rediseño del REA con el propósito de ir ajustar las actividades y su implementación a las necesidades educativas reales de los estudiantes.

\section{Referencias}

Butcher, N., Kanwar, A., \& Uvalic-Trumbic, S. (2015). Guía Básica de Recursos Educativos Abiertos (REA). Francia: UNESCO. Rescatado de https://unesdoc.unesco.org/ark:/48223/pf0000232986

Grugeon, Brigitte; Lagrange, Jean-Baptiste; Jarvis, Daniel; Alagic, Mara; Das, Mili y Hunscheidt, Diana (2010). Teacher Education Courses in Mathematics and Technology: Analyzing Views and Options. En: Celia Hoyles y Jean-Baptiste Lagrange (Eds.), Mathematics Education and Technology-Rethinking the Terrain, caplo 15, pp. 329-345. Springer US. DOI: 10.1007/978-1-4419-0146-0 15.

University of Colorado (2019). PhET: Free online physics, chemistry, biology, earth science and math simulations. Recuperado de https://phet.colorado.edu

Rubio-Pizzorno, Sergio. (2018). Integración digital a la práctica del docente de geometría. Tesis de Maestría no publicada. Ciudad de México, México: Centro de Investigaciones y de Estudios Avanzados (Cinvestav). DOI: 10.13140/RG.2.2.15488.94728/1.

Simon, Martin (1995). Reconstructing mathematics pedagogy from a constructivist perspective. Journal for Research in Mathematics Education, 26(2), pp. 114-145. ISSN 0021-8251. DOI: 10.2307/749205. 
VIII Congresso Brasileiro de Informática na Educação (CBIE 2019)

Anais dos Workshops do VIII Congresso Brasileiro de Informática na Educação (WCBIE 2019)

Swan, M. (2014). Design Research in Mathematics Education. En: Stephen Lerman (Ed.), Encyclopedia of Mathematics Education, pp. 148-152. Springer, Dordrecht. DOI: $10.1007 / 978-94-007-4978-8180$.

Téllez, R. (2019). Principio de Arquímedes [Libro GeoGebra]. Rescatado de geogebra.org/m/xytgyycg 